Voix et Images

voixetimages

\title{
Bibliographie de Roger Des Roches
}

\section{Roger Chamberland et André Gervais}

Volume 13, numéro 2 (38), hiver 1988

Le propre du corps Roger Des Roches

URI : https://id.erudit.org/iderudit/200710ar

DOI : https://doi.org/10.7202/200710ar

Aller au sommaire du numéro

Éditeur(s)

Université du Québec à Montréal

ISSN

0318-9201 (imprimé)

1705-933X (numérique)

Découvrir la revue

Citer ce document

Chamberland, R. \& Gervais, A. (1988). Bibliographie de Roger Des Roches. Voix et Images, 13(2), 280-289. https://doi.org/10.7202/200710ar d'utilisation que vous pouvez consulter en ligne.

https://apropos.erudit.org/fr/usagers/politique-dutilisation/ 


\section{Bibliographie de Roger Des Roches}

\section{Roger Chamberland, CRELIQ, Université Laval et André Gervais, Université du Québec à Rimouski}

Une bibliographie évidemment non exhaustive. Malgré les recherches des deux compilateurs et l'aide, à Radio-Canada, de quelques services que nous remercions chaleureusement. Des références aux travaux picturaux de l'auteur (couvertures de livres, maquettes de collections et de magazines, particulièrement) sont par ailleurs données dans l'entretien. (A.G.)

\section{Euvres}

Corps accessoires. Poèmes, Montréal, Éditions du Jour, [1970], 55 p., [reproduit dans] Tous, corps accessoires... (poèmes et proses), [Montréal], les Herbes rouges, [1979], 293 p. [v.p. 7-55].

L'Enfance d'yeux suivi de Interstice, Montréal, Éditions du Jour, [1972], 118 p., [reproduit dans] Tous, corps accessoires... (poèmes et proses), [Montréal], les Herbes rouges, [1979], 293 p. [v.p. 57-104 et 105-158].

Les Problèmes du cinématographe, les Herbes rouges, $\mathrm{n}^{\circ} 8$ (mars 1973), [31 p.], [reproduit dans] Tous, corps accessoires... (poèmes et proses), [Montréal], les Herbes rouges, [1979], 293 p. [v.p. 229-251].

SPACE-OPERA (sur-exposition), les Herbes rouges, $\mathrm{n}^{\circ} 15$ (décembre 1973) [28 p.], [reproduit dans] Tous, corps accessoires... (poèmes et proses), [Montréal], les Herbes rouges, [1979], 293 p. [v.p. 253-281].

Reliefs de l'Arsenal. Récit, [Montréal], l'Aurore, coll. «Écrire», [1974], 94 p., [reproduit avec quelques variantes dans] les Herbes rouges, $\mathrm{n}^{\circ} \mathrm{s}$ 145-146 (1986), 72 p.

La publicité discrète, les Herbes rouges, $\mathrm{n}^{\circ} 25$ (janvier 1975), $25 \mathrm{p}$.

Le Corps certain, les Herbes rouges, $\mathrm{n}^{\circ} 30$ (septembre 1975), $23 \mathrm{p}$.

Autour de Françoise Sagan indélébile. Poèmes et proses. 1969-1971, [Montréal], l'Aurore, [1975], 101 p., [reproduit dans] Tous, corps accessoires... (poèmes et proses), [Montréal], les Herbes rouges, [1979], 293 p. [v.p. 159-227].

La Vie de couple, les Herbes rouges, $\mathrm{n}^{\circ} \mathrm{s} 50-51$ (1977), [63 p.]. 
La Promenade du spécialiste, les Herbes rouges, $\mathrm{n}^{\circ} 54$ (1977), [23 p.].

Les Lèvres de n'importe qui, les Herbes rouges, $n^{\circ} 70$ (1978), 32 p.

Tous, corps accessoires... (poèmes et proses), [Montréal], les Herbes rouges, coll. «Enthousiasme», [1979], 293 p.

Avec Normand de Bellefeuille, Pourvu que ça ait mon nom, Montréal, les Herbes rouges, coll. «Lecture en vélocipède», $\mathrm{n}^{\circ} 23,1979,75$ p.

L'Observatoire romanesque, les Herbes rouges, $\mathrm{n}^{\circ} 77$ (1979), $31 \mathrm{p}$.

L'Imagination laïque, Montréal, les Herbes rouges, coll. «Lecture en vélocipède», $\mathrm{n}^{\circ} 29,1982,78 \mathrm{p}$.

Avec Marc Lafontaine, Bernard et Richard Angers, l'Écrivain public, système de traitement de texte pour Apple II et Commodore 64, Montréal, Logidisque, 1984.

Poème, attention! suivi de Deuxième Poème, les Herbes rouges, $\mathrm{n}^{\circ} 128$ (1984), $40 \mathrm{p}$.

Le Soleil tourne autour de la terre, Montreal, les Herbes rouges, 1985, 74 p.

Avec Francis Malka, l'Écrivain public - Action Writer, système bilingue de traitement de texte pour IBM PC, Montréal, Logidisque, 1987.

Tout est normal, tout est terminé, Montréal, les Herbes rouges, $\mathrm{n}^{\circ} 160$ (1987), $30 \mathrm{p}$.

II. Proses et poèmes publiés en revues et non repris en livre

«Toute leur anatomie complexe (cumul mixte)», $C u l Q, \mathrm{n}^{\circ} \mathrm{s}$ 4-5 (été-automne 1974), p. 81-84.

«Je sors d'une histoire d'amour et je suis épuisé», la Nouvelle Barre du jour, $n^{\circ} 64$ (mars 1978), p. 5-10.

«Les Sacrées Écoles», la Nouvelle Barre du jour, $\mathrm{n}^{\circ} 77$ (numéro intitulé $\grave{A}$ quelques obsessions près), avril 1979, p. 23-28.

«Les Faits réels (En guise d'introduction)», «Tous les veilleurs de nuit», «Et, après une nuit de veille» et «Sans titre», la Nouvelle Barre du jour, $\mathrm{n}^{\circ} \mathrm{s} 79$ 80 (numéros intitulés Science-fiction et préparés par Roger Des Roches et Louis-Philippe Hébert), juin 1979, p. 11-17, 105-109, 111-117 et 141-142.

«Quatre Nouveaux Articles définis», la Nouvelle Barre du jour, $\mathrm{n}^{\circ} \mathrm{s}$ 92-93 (numéros intitulés Poésie 1980), juin 1980, p. 115-120. 
«Un travail de circonstance», la Nouvelle Barre du jour, $\mathrm{n}^{\circ} \mathrm{S} 122-123$ (numéros intitulés Écritures 1983), février 1983, p. 100-104.

«La Sortie», la Nouvelle Barre du jour, $\mathrm{n}^{\circ} 140$ (numéro intitulé Sortie lexit), juin 1984, p. 58.

«Célibataire», la Nouvelle Barre du jour, n 143 (numéro intitulé l'Écriture célibataire), novembre 1984, p. 51-62.

III. Articles et critiques

\section{A) Littérature}

Avec François Charron, «Notes sur une pratique», la Barre du jour, $\mathrm{n}^{\circ} 29$ (été 1971), p. 2-7.

«Incursion dans un lieu neutre. La critique littéraire sans histoire», Presqu'Amérique, Québec, vol. 1, nº 8 (août 1972), p. 31-33.

«Incursions dans un lieu neutre», Presqu'Amérique, vol. 1, n 9 (septembre octobre 1972), p. 28-30.

«En un texte décousu, 1138 mots sur Patrick Straram, ce qui est fort peu», Hobo-Québec, $\mathrm{n}^{\circ} \mathrm{s}$ 9-11 (numéros intitulés Spécial Straram), octobrenovembre 1973, p. 13.

«De l'importance inestimable pour l'adolescent de lectures saines et constructives (préface fortement anecdotique)», préface à la réédition de Denis Vanier, Je, l'Aurore, coll. «Lecture en vélocipède», [novembre] 1974, p. 11-18.

«Space-operas. première partie: les mouvements rythmés de la pénétration», Hobo-Québec, $\mathrm{n}^{\circ} 22$ (mars-avril 1975), p. 14-16.

«(Entre autres) comment mes textes m'ecrivent», Chroniques, vol. 1, n's 6-7 (numéros intitulés les Conditions de l'écriture), juin-juillet 1975, p. 41-46.

«Un deuil fébrile», Spirale, n 1 (septembre 1979), p. 11.

«Et si nous étions seuls?», Spirale, $\mathrm{n}^{\circ} 3$ (novembre 1979), p. 10.

«Une écriture sans délire», Spirale, $\mathrm{n}^{\circ} 4$ (décembre 1979), p. 10.

«Le Doute royal», Spirale, $n^{\circ} 5$ (janvier 1980), p. 1.

«Pour les Nouveaux Écrivains», le Devoir, 22 mars 1980, p. 24.

«La Nuit de Walpurgis», Spirale, $\mathrm{n}^{\circ} 9$ (mai 1980), p. 3. 
«La Vie de château», Spirale, $\mathrm{n}^{\circ} 12$ (octobre 1980), p. 1

«La Sainte Horreur», Spirale, $\mathrm{n}^{\circ} 17$ (mars 1981), p. 4.

«La Parole et la résistance», Spirale, $\mathrm{n}^{\circ} 20$ (juin 1981), p. 4-5.

B) Musique rock et cinéma

«Annexe à une critique de Soublière, mais, comme toujours, ben straight, alors que quelque part dans ma chambre $\rightarrow$, Hobo-Québec, $\mathrm{n}^{\circ} \mathrm{s} 14-15$ (janvier 1974), p. 24 [la chronique de Roger Soublière est parue dans les numéros 12-13, décembre 1973, p. 28-29].

«Patti Smith vague?», Spirale, nº 1 (septembre 1979), p. 15.

«Les Effets, trop d'effets», Spirale, n 4 (décembre 1979), p. 16.

«Le Scientifique au poste de commande», Spirale, $\mathrm{n}^{\circ} 7$ (mars 1980), p. 8-9.

«Never listen to electric guitar», Spirale, $\mathrm{n}^{\circ} 8$ (avril 1980), p. 8.

«Teenybopper un jour, teenybopper toujours» et «Les Morts vont vite», Spirale, $\mathrm{n}^{\circ} 13$ (novembre 1980), p. 15 et 16.

«Un vieil homme indigne», Spirale, $\mathrm{n}^{\circ} 18$ (avril 1981), p. 13.

«Personne n'écoute les paroles», Spirale, nº 19 (mai 1981); p. 14.

«Rock qui trouble, rock qui gêne» et «Quand la performance a du bon», Spirale, $\mathrm{n}^{\circ} 21$ (septembre 1981), p. 8-9 et 18.

«Oublier les voix du passé», Spirale, $\mathrm{n}^{\circ} 22$ (février 1982), p. 11.

IV. Textes pour la radio (CBF-MF)

«La Nouvelle Moraie interplanétaire», «la Distance incertaine», «Des autres» et «la Pharmacopée de l'espace», $1^{\mathrm{re}}, 2^{\mathrm{e}}, 4^{\mathrm{e}}$ et $5^{\mathrm{e}}$ émissions (d'une heure chacune) d'un ensemble de 6 documentaires dramatiques intitulé $A u$-delà de la science: fiction et réalisé par Louis-Philippe Hébert (14 septembre, 21 septembre, 5 octobre et 12 octobre 1978); lecteurs: Louise Turcot et Ronald France [les deux autres émissions ont été écrites par Germain Beauchamp].

«L'Autobus», récit, l'Atelier des inédits (série d'émissions de 30 minutes chacune, réalisée par Raymonde Fafard), 14 novembre 1979; lectrice: Ghislaine Paradis. 
«La Matinée de l'épisode», récit, Alternances (série d'émissions de 30 minutes chacune, réalisée par Raymond Fafard), 17 juin 1981; lecteur: Luc Durand.

«Derrière quelques masques, la ressemblance», récit, Alternances, 16 mai 1982; lectrice: Marie Eykel.

«La Plus petite étoile», dramatique, la Feuillaison (série d'émissions de 30 minutes chacune, réalisée par Claude Godin), 11 juin 1982.

«Célibataire», récit, Alternances, 18 mars 1984; lecteur: Normand Chouinard.

«La Princesse radiophonique», adaptation d'un texte publié dans Autour de..., Éloges de la radio (série d'émissions de 30 minutes chacune, réalisée par Aline Legrand), 5 novembre 1985; lecteur: Ronald France.

«Le Roi de cœun», adaptation d'une nouvelle d'Ed McBain, et «Votre dévoué Jack l'Éventreur», adaptation d'une nouvelle de Robert Bloch, Énigmes et fiction (série d'émissions de 30 minutes chacune, réalisée par Lucie Ménard), 3 juillet et 17 juillet 1986; lecteurs: Jean Marchand pour la première, Benoît Dagenais et Pierre Collin pour la seconde.

\section{Entrevues à la radio (CBF-MF)}

Avec Jean Sarrazin: à propos de l'Enfance d'yeux suivi de Interstice, Carnet Arts et lettres, 21 avril 1972.

Avec Réginald Martel et Jacques Godbout: à propos de l'Enfance d'yeux suivi de Interstice, Book-club, 24 avril 1972.

Avec Louis-Philippe Hébert: à propos de sa conception de la poésie, l'État de la jeune poésie, 5 juin 1978.

Avec Madeleine Champagne: à propos du colloque de Cerisy sur la littérature québécoise, Dossiers, 7 mars 1981.

\section{Conférences}

«Le Métier et les pièges», donnée à l'occasion du colloque la Nouvelle Écriture, Montréal, février 1980; la Nouvelle Barre du jour, nº 90-91, mai 1980, p. $153-160$.

«Le Métier, les maîtrises et les p'tites histoires», donnée à l'occasion du colloque Littérature québécoise d'aujourd'hui: situations et formes, Cerisy-la-Salle (France), août 1980, inédite.

«Écriture et modernité», donnée à l'occasion du colloque l'Écriture de la modernité, Montréal, septembre 1981, inédite. 
«Les Lettres de démission», donnée à l'occasion du colloque la Littérature québécoise. Des origines à nos jours, Sainte-Adèle, octobre 1983, inédite.

«Les Écrivains et le traitement de texte», donnée à l'occasion du colloque Écriture et informatique, Sherbrooke, novembre 1983, inédite.

«L'Édition de logiciels», donnée à l'occasion du stage d'édition organisé par la Société de développement du livre et dupériodique, Saint-Marc-sur-Richelieu, novembre 1983, inédite.

«Le Logiciel entre l'industrie et la culture», donnée à l'occasion du colloque la Place du Québec dans l'industrie du logiciel, Montréal, janvier 1985, inédite.

«L'Informatique et l'écriture», donnée dans le cadre des activités du Salon du livre de Québec, avril 1986, inédite.

VII. Études sur les œuvres

Sur l'œuvre en général

CHAMBERLAND, Roger, «Corps accessoires et autres recueils de poésies et récits de Roger Des Roches», Dictionnaire des œuvres littéraires du Québec, tome V, 1970-1975, Montréal, Fides, 1987, p. 186-190.

\section{Sur Corps accessoires}

[ANONYME], «Corps...», le Livre canadien, vol. 1, n² 202 (1970).

BASILE, Jean, «Aux Éditions du Jour. Publications de trois "jeunes poètes"», le Devoir (29 septembre 1970), p. 12.

LAVOIE, Philippe, «Corps...», Feuillets des jeunesses littéraires du Québec, vol. 17 (octobre 1970), p. 7-8.

MARTEL, Réginald, «Trois Poètes: un trèfle à quatre feuilles», la Presse (3 octobre 1970), p. C-2.

TOURIGNY, François, «Interlignes», le Trait d'union [Journal des étudiants du CEGEP de Maisonneuve], vol. 31, $\mathrm{n}^{\circ} 2$ (12 octobre 1970), p. 11.

PILON, Jean-Guy, «Une jeune poésie de commencement», le Devoir (7 novembre 1970), p. 11.

DIONNE, René, «Corps...», Relations, n 357 (février 1971), p. 55.

FOURNIER, Gérard-Claude, «Trois Poètes», Livres et auteurs québécois 1970, Québec, PUL, 1971, p. 147. 


\section{Sur l'Enfance d'yeux / Interstice}

PARADIS, Suzanne, «Des Roches, Beausoleil, Lavergne, Bernier et Paré. La jeune poésie québécoise après la nuit de la poésie», le Soleil (26 août 1972), p. 47.

BLAIS, Jacques, «l'Enfance...», University of Toronto Quarterly, vol. $62, \mathrm{n}^{\circ} 4$ (Summer 1973), p. 383.

\section{Sur les Problèmes du cinématographe}

STRARAM, Patrick, «Écritures en situation. Les Problèmes du cinématographe», Hobo-Québec, ns 5-7 (été 1973), p. 11, 43.

PILON, Jean-Guy, «les Mini-maisons», le Devoir (18 août 1973), p. 13.

HAECK, Philippe, «la Circulation des lettres. Les Herbes rouges polysèment», Hobo-Québec, nº 14-15 (janvier 1974), p. 23.

CARRIÈRE, André, «Trois Auteurs en quête d'un langage. Pierre Milot. Louis Geoffroy. Roger Des Roches», Livres et auteurs québécois 1973, Québec, PUL, 1974, p. 100-101.

\section{Sur Reliefs de l'Arsenal}

BEAUSOLEIL, Claude, «Reliefs de l'Arsenal: une écriture d'avant-garde», $C U L Q, n^{\circ}$ s 4-5 (été-automne 1974), p. 85-91.

—, «Reliefs...»,Hobo-Québec, nº 21 (janvier-février 1975), p. 31.

HAECK, Philippe, «Reliefs...», Chroniques, vol. 1, $\mathrm{n}^{\circ} 1$ (janvier 1975), p. 4748.

VIDAL, Jean-Pierre, «Roger Des Roches. Reliefs de l'Arsenal», Livres et auteurs québécois 1974, Québec, PUL, 1975, p. 68-69.

CIMON, Renée, «Reliefs...», Nos livres, vol. 8, n 388, décembre 1977.

CHASSAY, Jean-François, «Reliefs...», Spirale, $\mathrm{n}^{\circ} 64$ (octobre 1986), p. 5.

\section{Sur la Publicité discrète}

BEAUSOLEIL, Claude, «Lire aujourd'hui. Changement, désir, plaisir», HoboQuébec, ns 23-24 (mai-août 1975), p. 5.

HAECK, Philippe, «la Publicité...», Chroniques, vol. 1, ns 6-7 (juin-juillet 1975), p. 129-130. 
-, «la Poésie. Roger Des Roches et les 'Corps fantômes'», le Devoir (22 août 1975), p. 12.

JACQUES, Réjean, «Changer les mots et la politique», la Presse (5 juillet 1975), p. D-4.

GIGUÈRE, Richard, «la Publicité discrète et le Corps incertain», Livres et auteurs québécois 1975, Québec, PUL, 1976, p. 120-121.

Sur Autour de Françoise Sagan indélébile

HAECK, Philippe, «la Poésie. Roger Des Roches et les 'Corps fantômes'», le Devoir (22 août 1975), p. 12.

—, «Autour de...», Chroniques, $\mathrm{n}^{\circ} 15$ (mars 1976), p. 43-45.

[ANONYME], «Autour de...», le Livre canadien, $\mathrm{n}^{\circ}$ 335, novembre 1975.

AUDET, Noël, «Autour de...», Voix \& images, vol. 1, n 2 (décembre 1975), p. $289-290$.

Sur Tous, corps accessoires...

BEAUSOLEIL, Claude, «Tous,...», Livres et auteurs québécois 1979, Québec, PUL, 1980, p. 110-114.

—, «Roger Des Roches: un novateur», le Devoir, (23 février 1980), p. 26.

NEPVEU, Pierre, «les Années soixante-dix: du commencement à la fin», Lettres québécoises, $\mathrm{n}^{\circ} 18$, (printemps 1980), p. 26-29.

CHAMBERLAND, Roger, «Tous,...», Québec français, $\mathrm{n}^{\circ} 40$ (décembre 1980), p. 15.

Sur les Lèvres de n'importe qui

CORRIVEAU, Hugues, «Poésie: des lèvres et des vertiges», la Nouvelle Barre $d u$ jour, $\mathrm{n}^{\circ} 8$ (septembre 1979), p. 87-89

BELLEFEUILLE, Normand de, «Ouverture pour une lettre d'amour: les Lèvres de n'importe qui», Spirale, $\mathrm{n}^{\circ} 1$ (septembre 1979), p. 8.

GIGUÈRE, Richard, «les Lèvres...», Livres et auteurs québécois 1979, Québec, PUL, 1980, p. 105. 


\section{Sur l'Observatoire romanesque}

BEAUSOLEIL, Claude, «Quelques confidences et plaisirs», le Devoir (31 mai 1980), p. 19.

M.B., «les Herbes rouges. À la défense des tendances les plus avant-gardistes», l'Union des Cantons de l'Est (18 novembre 1980).

Sur Pourvu que ça ait mon nom

BEAUSOLEIL, Claude, «Quelques confidences et plaisirs», le Devoir (31 mai 1980), p. 19.

AQUIN, Pierre-Stéphane, «Pourvu que...», le Bulletin Pantoute, $\mathrm{n}^{\circ} 3$ (septembre-novembre 1980), p. 8.

NEPVEU, Pierre, «De 'l'importance de la littérature'», Lettres québécoises, $n^{\circ} 19$ (automne 1980), p. 30.

\section{Sur l'Imagination laique}

LABINE, Marcel, «Contre la théorie, l'imagination», Spirale, $\mathrm{n}^{\circ} 29$ (novembre 1982), p. 9.

CORRIVEAU, Hugues, «l'Imagination...», Livres et auteurs québécois 1982, Québec, PUL, 1983, p. 105-107.

MARTIN, Raymond, «l'Imagination...», Mcebius, $\mathrm{n}^{\circ} 17$ (printemps 1983), p. $90-91$.

BAYARD, Caroline, «Letters in Canada. Livres en français. La poésie», University of Toronto Quarterly, vol. 52, n 4 (Summer 1983), p. 359-361.

BOUCHARD, Christian, «Du muscle et de l'intelligence. L'Imagination laïque», Lettres québécoises, $\mathrm{n}^{\circ} 30$ (été 1983), p. 26-28.

BROCHU, André, «De la mer à la mer», Voix \& images, vol. $9, \mathrm{n}^{\circ} 1$ (automne 1983), p. 145-146.

D'ALFONSO, Antonio, «l'Imagination...», Nos livres, vol. 14 (octobre 1983), p. 19-20.

\section{Sur l'Écrivain public}

[ANONYME], «De la page blanche à l'écran noir», le Devoir (26 janvier 1985), p. 23. 
MARTEL, Réginald, «Roger Des Roches et 'lÉcrivain public'. Les puces et la littérature», la Presse (2 février 1985), p. E-4.

CAUCHON, Paul, «Micro Mag» (mars 1985), p. 26, 28-30.

ROYER, Jean, «'Un petit Mozart de la programmation’. Francis Malka et 'l'Écrivain public'», le Devoir (3 octobre 1987), p. D-1, D-8.

Sur Poème, attention! suivi de Deuxième Poème

DESGENT, Jean-Marc, «Poème, attention!...», Nos livres, vol. 16 (mars 1985), p. 24-25.

BROCHU, André, «Des fous et des autres», Voix \& images, vol. $\mathrm{X}, \mathrm{n}^{\circ} 3$ (printemps 1985), p. 184.

\section{Sur le Soleil tourne autour de la terre}

NEPVEU, Pierre, «Poèmes d'air ou de matière», Spirale, $\mathrm{n}^{\circ} 59$ (mars 1986), p. 4.

TOUPIN, Gilles, «Explorer l'âme, le cœur, le cul en deux temps, trois mouvements (p. 58)», la Presse (28 juin 1986), p. E-2.

MARQUIS, André, «Aux Herbes rouges: détresse et violence», Lettres québécoises, nº 42 (été 1986), p. 36-37.

BROCHU, André, «Lascaux, les limbes et autres lieux», Voix \& images, vol. XI, nº 4 (août 1986), p. 134-135.

D'ALFONSO, Antonio, «le Soleil...», Nos livres, vol. 17 (octobre 1986), p. 36-37. 